\title{
MOLECULAR DYNAMICS IN SOLUTION PROBED BY THE TRANSIENT GRATING METHOD WITH A NANOSECOND PULSED LASER
}

\author{
MASAHIDE TERAZIMA*, KOICHI OKAMOTO, NOBORU HIROTA \\ Department of Chemistry, Faculty of Science, Kyoto University, Kyoto, 606 Japan
}

(Received 5 March, 1993)

\begin{abstract}
Molecular dynamics in organic solution is investigated by the transient grating method with a nanosecond pulsed laser and a fast detection system. First, the dynamics of methyl red (MR) is studied for the probe of the translational diffusion process of solution. The origin of the TG signal which represents the mass diffusion is attributed to the phase grating created by the different refractive indices of the cis and trans forms. The cis form of MR, which has a slightly smaller molecular volume than the trans form is found to diffuse more slowly in alcoholic and aldehyde solutions. The measured diffusion coefficients (D) are compared with theoretically calculated values. Second, the TG method is applied to elucidate the dynamics of transient radicals created by photochemical reactions. The determined $\mathrm{D}$ of the radicals are about 3 4 times smaller than those of the parent non-radical molecules even though the molecular volumes are similar to each other. The advantages as well as the disadvantages of the TG method for elucidating the solvent dynamics are discussed.
\end{abstract}

KEY WORDS: transient grating, diffusion, methylred, photochemical reaction

\section{INTRODUCTION}

The transient grating (TG) technique is one of nonlinear optical spectroscopies in which four photons are involved. ${ }^{1}$ Phenomenologically, this method can be understood as the probe beam diffraction by a grating created by the interference of two excitation beams (Figure 1). Depending on the nature of the grating, one can optically probe various kinds of dynamics, such as intramolecular and intermolecular energy flow dynamics, ${ }^{2}$ free carrier (electron or hole) dynamics, ${ }^{3}$ viscoelastics (structural relaxation), ${ }^{4}$ rotation or orientational motions, ${ }^{5}$ dynamics of chemical reactions ${ }^{6}$ and so on with a very wide time range from a order of second to femtosecond.

If we are interested in solution dynamics, this TG method will be a very useful and powerful way for studying the translational molecular motions in solution. The principle of the measurement is as follows. If the sample contains photochemically active molecules, these molecules are converted to other species in the bright region of the grating but not in the dark region. This site selective excitation induces a

\footnotetext{
* To whom correspondence should be addressed.
} 

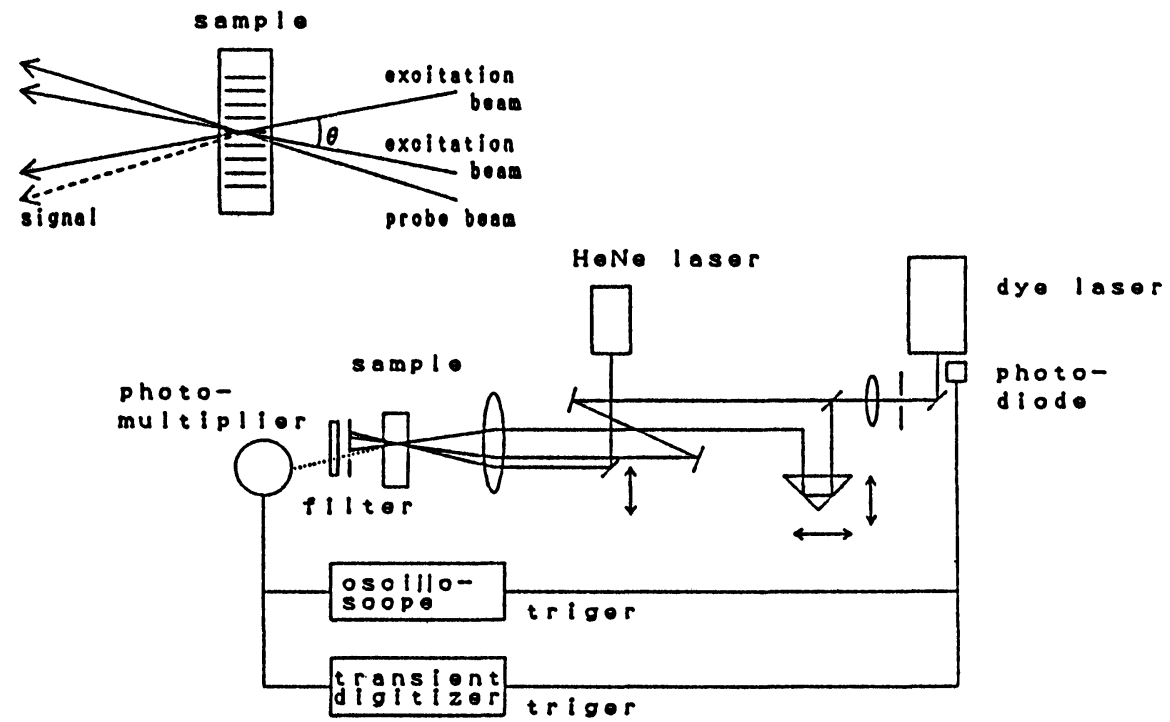

Figure 1 Experimental setup for the TG measurement and the configuration of the excitation and probe beams.

spatial modulation of refractive index and/or absorption coefficient. The grating diffracts another probe beam entering in this region. The signal decays as the grating smears out by the diffusion. Therefore, the decay of the TG signal reflects the diffusion process of the probe molecule. The TG method has several unique advantages.

1. This method possesses very high sensitivity, because the TG signal is detected without background light.

2. Since the grating is created in a very small space in a sample, only a very small amount of the sample is required for the signal detection.

3. We monitor the smear-out time of the very narrow-spacing fringes by diffusion. Therefore, even in a very viscous solution, it takes only a short time to complete one experiment. Also, because of the very fast response time of the detection system, it can follow very fast diffusion. As a result, a wide range of diffusion coefficient, $\mathrm{D}\left(10^{-7} \sim 10^{-15} \mathrm{~m}^{2} \mathrm{~s}^{-1}\right)$ can be measured without difficulty.

4. By choosing the direction of the fringe against a specific axis of the sample, anisotropy of the diffusion can be investigated.

5. Several methods which utilize the transient effects of quenching processes have been developed to study the diffusion of transient species, ${ }^{7}$ but these methods are usually based on some assumptions on the quenching process. The TG method directly observes the diffusional motion of the molecule in space.

6. If we use a pulsed laser for the excitation light, the time response can be very fast. It will be possible to study a diffusion process of a very short-lived transient species. 
7. If we vary the fringe spacing, $\Lambda$, the species should move various distance before smearing out the grating. This procedure has been used for discriminating the diffusion process from a natural decaying process of the unstable species..$^{8-11}$ More interestingly, this $\Lambda$ dependent TG measurement will give an opportunity to find an anomalous diffusion process, which shows a distance dependent diffusion coefficient as will be discussed in section 4.3.

So far, the TG studies on diffusion processes have been mainly concerned with dye diffusion in polymer solutions, ${ }^{8}$ polymer glasses, ${ }^{9}$ a gel ${ }^{10}$ and liquid crystals, ${ }^{11}$ because it is less time consuming and, therefore, makes more accurate measurements possible compared with the traditional methods (advantage 3). In this paper, we first describe the solution dynamics studied by the TG method with methyl red (MR). With a pulsed laser and a fast detection system, we are able to study fast molecular dynamics in normal organic solution after the decay of the thermal grating signal. From the analysis of the time dependence of the TG signal, the origin of the signal which represents the mass diffusion of MR is attributed to the phase grating. The diffusion coefficients of the cis and trans isomers are discussed. Next, we apply the TG method to measure D of unstable transient radicals created by photochemical reactions in solution. We find that the radicals created by the photoinduced hydrogen abstraction reactions usually have much smaller $D$ than those of the parent molecules even though the molecular volumes do not differ so much. Finally, we discuss the above mentioned advantages as well as the disadvantages of the TG method.

\section{EXPERIMENT}

Transient grating is created by the interference of two excitation beams from an excimer laser-pumped dye laser (Lumonics Hyper 400, Lumonics Hyper Dye 300) for the photoexcitation of MR. The excimer laser light is directly used for the measurement of $\mathrm{D}$ of transient radicals. The repetition rate of the excitation pulse was typically $\sim 3 \mathrm{~Hz}$. A He-Ne laser was brought into the crossing region at the Bragg condition for the probe beam. The crossing angle was first roughly estimated by measuring the distance between the two excitation beams and that between the focus lens and the crossing point (Figure 1). We then determined $\mathrm{q}^{2}$ more accurately at each crossing angle from the decay rate constant of the thermal grating signal $\left(\mathrm{k}_{\mathrm{th}}\right)$ and $\mathrm{D}_{\text {th }}$ to correct a small error in measuring the crossing angle. The diffracted signal was detected by a photomultiplier (Hamamatsu R928) and averaged by a digital oscilloscope (Tektronix 2430A) and a microcomputer. The relaxation time was measured as a function of the crossing angle.

Methyl red was purified by recrystallization. Pyrazine was purified by vacuum sublimation. Spectrograde solvents were used without further purification. Concentration of MR was $7.4 \times 10^{-4} \mathrm{M}$. For the measurement of D of the radicals, fresh samples were used after every $\sim 500$ shot irradiation to avoid the effect of reaction products. 


\section{METHOD}

The grating is created in a sample solution by crossing two coherent excitation beams with an angle $\theta^{1}$. Due to the interference pattern of these beams, the light intensity in the crossing region is modified as

$$
\mathrm{I}(\mathrm{x})=\frac{I}{2}(1+\cos (2 \pi \mathrm{x} / \Lambda))
$$

where $\mathrm{I}$ is the power of the excitation laser and the fringe spacing, $\Lambda$, is given by

$$
\Lambda=\frac{\lambda e x}{2 \sin (\theta / 2)}
$$

and $\lambda_{\mathrm{ex}}$ is the wavelength of the excitation light. Here we assume that the TG dynamics we are interested in occurs in a sufficiently longer time scale than the pulse width of the excitation beam. In proportion to the light intensity $\mathrm{I}(\mathrm{x})$ in the crossing region, molecules are excited to the excited states in which photo-isomerization (MR) or photochemical reactions take place. Since the created species usually have different optical properties (such as absorption coefficients and refractive indices), the spatially modulated photoexcitation induces a spatially modulated absorbance (amplitude grating) or refractive index (phase grating) at the probe beam wavelength. The refractive index and absorption coefficient in the sample solution after the excitation are represented by

$$
\begin{gathered}
\mathrm{n}(\mathrm{x}, \mathrm{t})=\mathrm{n}_{0}+\delta \mathrm{n}_{\mathrm{p}}(\mathrm{x}, \mathrm{t})+\delta \mathrm{n}_{\mathrm{th}}(\mathrm{x}, \mathrm{t}) \\
\mathrm{k}(\mathrm{x}, \mathrm{t})=\mathrm{k}_{0}+\delta \mathrm{k}_{\mathrm{p}}(\mathrm{x}, \mathrm{t})
\end{gathered}
$$

where $\mathrm{n}_{0}$ and $\mathrm{k}_{0}$ are, respectively, the unperturbed refractive index and absorption coefficient of the solution. The contribution of $\delta n_{t h}(x, t)$ represents the refractive index change by the thermal expansion of the solution due to the unavoidable radiationless transition from the excited state and it is given by

$$
\delta \mathrm{n}_{\mathrm{th}}(\mathrm{q}, \mathrm{t})=\mathrm{CI} \delta \mathrm{n}_{\mathrm{th}}^{0} \exp \left(-\mathrm{q}^{2} \mathrm{D}_{\mathrm{th}} \mathrm{t}\right)
$$

where $\mathrm{C}$ is a proportional constant, $\mathrm{D}_{\mathrm{th}}$ is the thermal diffusion coefficient, and $\delta \mathrm{n}_{\mathrm{th}}(\mathrm{q}, \mathrm{t})$ is the spatial Fourier component of $\delta \mathrm{n}_{\mathrm{th}}(\mathrm{x}, \mathrm{t})(\mathrm{q}=2 \pi / \Lambda)$. Since the refractive index decreases with increasing temperature, $\delta \mathrm{n}_{\mathrm{th}}^{0}$ should be negative. The other terms, $\delta \mathrm{n}_{\mathrm{p}}$ and $\delta \mathrm{k}_{\mathrm{p}}$, come from the spatially modulated distribution of chemical species (the subscript $\mathrm{p}$ stands for 'the population grating'). Under the thick grating and the weak diffraction conditions, the TG signal intensity $\mathrm{I}_{\mathrm{TG}}(\mathrm{t})$ at a grating vector $\mathrm{q}$ is represented by ${ }^{1}$

$$
\mathrm{I}_{\mathrm{TG}}(\mathrm{t})=\mathrm{A}\left(\delta \mathrm{n}_{\mathrm{th}}(\mathrm{q}, \mathrm{t})+\delta \mathrm{n}_{\mathrm{p}}(\mathrm{q}, \mathrm{t})\right)^{2}+\mathrm{B}\left(\delta \mathrm{k}_{\mathrm{p}}(\mathrm{q}, \mathrm{t})\right)^{2}
$$


(a) TG signal after the photoexcitation of $M R$

After the photoexcitation of trans-MR, which is a stable form in the ground state at room temperature, it is converted to the cis form and the cis form is gradually converted back to the trans form in dark.
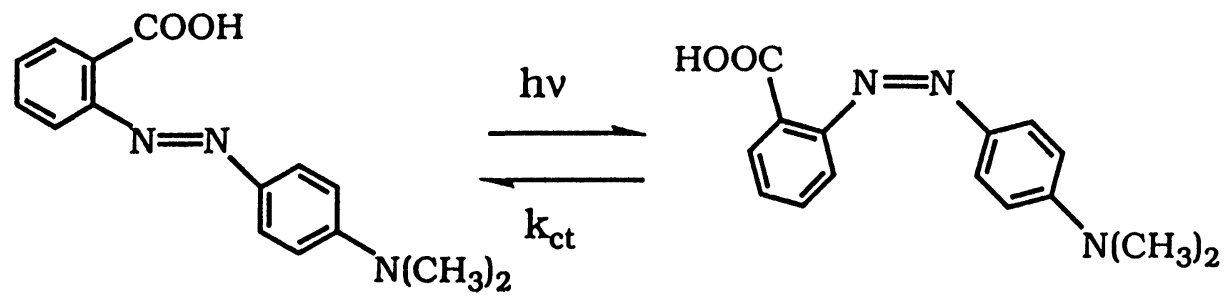

By solving the thermal and mass diffusion equations including the back photochemical process from cis to trans with the rate constant $k_{\mathrm{c}}$, Fourier components of both terms, $\delta \mathrm{n}_{\mathrm{p}}(\mathrm{q}, \mathrm{t})$, and $\delta \mathrm{k}_{\mathrm{p}}(\mathrm{q}, \mathrm{t})$ are given by, ${ }^{12}$

$$
\begin{gathered}
\delta \mathrm{n}_{\mathrm{p}}(\mathrm{q}, \mathrm{t})=\delta \mathrm{n}_{1} \exp \left(-\mathrm{D}_{\mathrm{t}} \mathrm{q}^{2} \mathrm{t}\right)+\delta \mathrm{n}_{2} \exp \left(-\left(\mathrm{D}_{\mathrm{c}} \mathrm{q}^{2}+\mathrm{k}_{\mathrm{c}}\right) \mathrm{t}\right) \\
\delta \mathrm{k}_{\mathrm{p}}(\mathrm{q}, \mathrm{t})=\delta \mathrm{k}_{1} \exp \left(-\mathrm{D}_{\mathrm{t}} \mathrm{q}^{2} \mathrm{t}\right)+\delta \mathrm{k}_{2} \exp \left(-\left(\mathrm{D}_{\mathrm{c}} \mathrm{q}^{2}+\mathrm{k}_{\mathrm{c}}\right) \mathrm{t}\right)
\end{gathered}
$$

and

$$
\begin{gathered}
\delta n_{1}=-[\Delta c] \delta n_{t} \frac{D_{c} q^{2}-D_{t} q^{2}}{D_{c} q^{2}-D_{t} q^{2}+k_{c t}} \\
\delta n_{2}=[\Delta c]\left(\delta n_{c}-\delta n_{t} \frac{k_{c t}}{D_{c} q^{2}-D_{t} q^{2}+k_{c t}}\right) \\
\delta k_{1}=-[\Delta c] \delta k_{t} \frac{D_{c} q^{2}-D_{t} q^{2}}{D_{c} q^{2}-D_{t} q^{2}+k_{c t}} \\
\delta k_{2}=[\Delta c]\left(\delta k_{c}-\delta k_{t} \frac{k_{c t}}{D_{c} q^{2}-D_{t} q^{2}+k_{c t}}\right)
\end{gathered}
$$

where subscripts $\mathrm{c}$ and $\mathrm{t}$ represent the cis and trans forms, respectively for the corresponding properties. $[\Delta c]$ is the concentration change of the cis form by the photo-excitation. Here, we have neglected a coupling term between the thermal and mass diffusion, which is usually very small in the organic solvents we examined at room temperature. Since the peaks of the absorption spectra of the cis and trans forms are located at shorter wavelengths than that of the He-Ne laser, both of $\delta n_{c}$ and $\delta n_{t}$ should be positive. 
(b) TG signal of photochemical reaction systems

In the case of photochemical reactions, usually more species are involved in the created grating. For example, we consider the following photoinduced hydrogen abstraction reaction from $\mathrm{AH}$ by a molecule $\mathrm{M}$ in the excited triplet state to create transient radicals $\mathrm{A} \cdot$ and $\mathrm{MH}$.

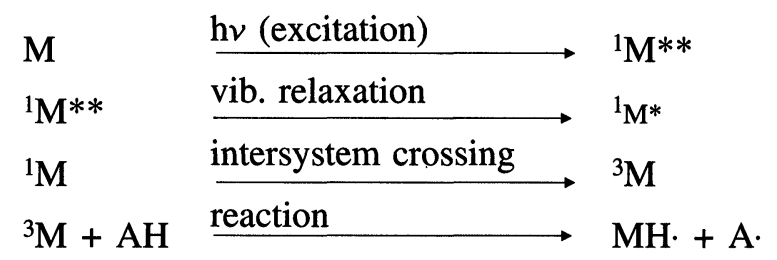

The radicals $\mathrm{MH}$. and $\mathrm{A} \cdot$ will disappear by subsequent reactions. After solving the diffusion equations of species $i(i=M H \cdot$ and $A \cdot)$ and $j(j=M$ and $A H)$, the concentration of each species is given by

$$
\begin{gathered}
C_{i}(q, t)=D I C_{0} \exp \left(-\left(D_{i} q^{2}+k_{i}\right) t\right) \\
C_{j}(q, t)=D I C_{0} \exp \left(-D_{j} q^{2} t\right)
\end{gathered}
$$

where $\mathrm{D}$ is a proportional constant, $\mathrm{C}_{0}$ is the initial concentration of the photoreactive molecule and we assume that the subsequent reactions are expressed by the first order reaction rate constant $\mathrm{k}_{\mathrm{i}}$. If the reactions are not the first order, the time dependence should be modified. The refractive index and the absorption coefficient accompanied with these species are given by

$$
\begin{aligned}
& \delta \mathrm{n}_{\mathrm{p}}(\mathrm{q}, \mathrm{t})=\operatorname{DIC}_{0}\left\{\Sigma \delta \mathrm{n}_{\mathrm{i}}^{0} \exp \left(-\left(\mathrm{D}_{\mathrm{i}} \mathrm{q}^{2}+\mathrm{k}_{\mathrm{i}}\right) \mathrm{t}\right)-\Sigma \delta \mathrm{n}_{\mathrm{j}}^{0} \exp \left(-\mathrm{D}_{\mathrm{j}} \mathrm{q}^{\mathrm{t}} \mathrm{t}\right)\right\} \\
& \delta \mathrm{k}_{\mathrm{p}}(\mathrm{q}, \mathrm{t})=\operatorname{DIC}_{0}\left\{\Sigma \delta \mathrm{k}_{\mathrm{i}}^{0} \exp \left(-\left(\mathrm{D}_{\mathrm{i}} \mathrm{q}^{2}+\mathrm{k}_{\mathrm{i}}\right) \mathrm{t}\right)-\Sigma \delta \mathrm{k}_{\mathrm{j}}^{0} \exp \left(-\mathrm{D}_{\mathrm{j}} \mathrm{q}^{2} \mathrm{t}\right)\right\}
\end{aligned}
$$

In order to separate out the diffusion process from the intrinsic decay of the radical or the cis form of MR in the TG signal decay, the TG signal is measured as a function of $\mathrm{q}^{2}$.

In summary, the time dependence of the TG signal after the photoexcitation of MR and the photoreactive molecule $M$ are interpreted by the eqs. (5), (6) and (8).

\section{RESULTS AND DISCUSSION}

\subsection{Diffusion of MR in nonviscous organic solutions}

Although a dye molecule, MR has been extensively used ${ }^{8-a-e, 9-a-b, 10,11}$ for studying the dynamics in viscous solution, the character of the TG signal of MR in ordinary solution has not been studied. In order to apply this dye to probe the diffusion dynamics, however, it is desirable to know the fundamental properties of the TG signal in ordinary solutions of small molecules. ${ }^{12}$ Figure 2 and 3 show the time 

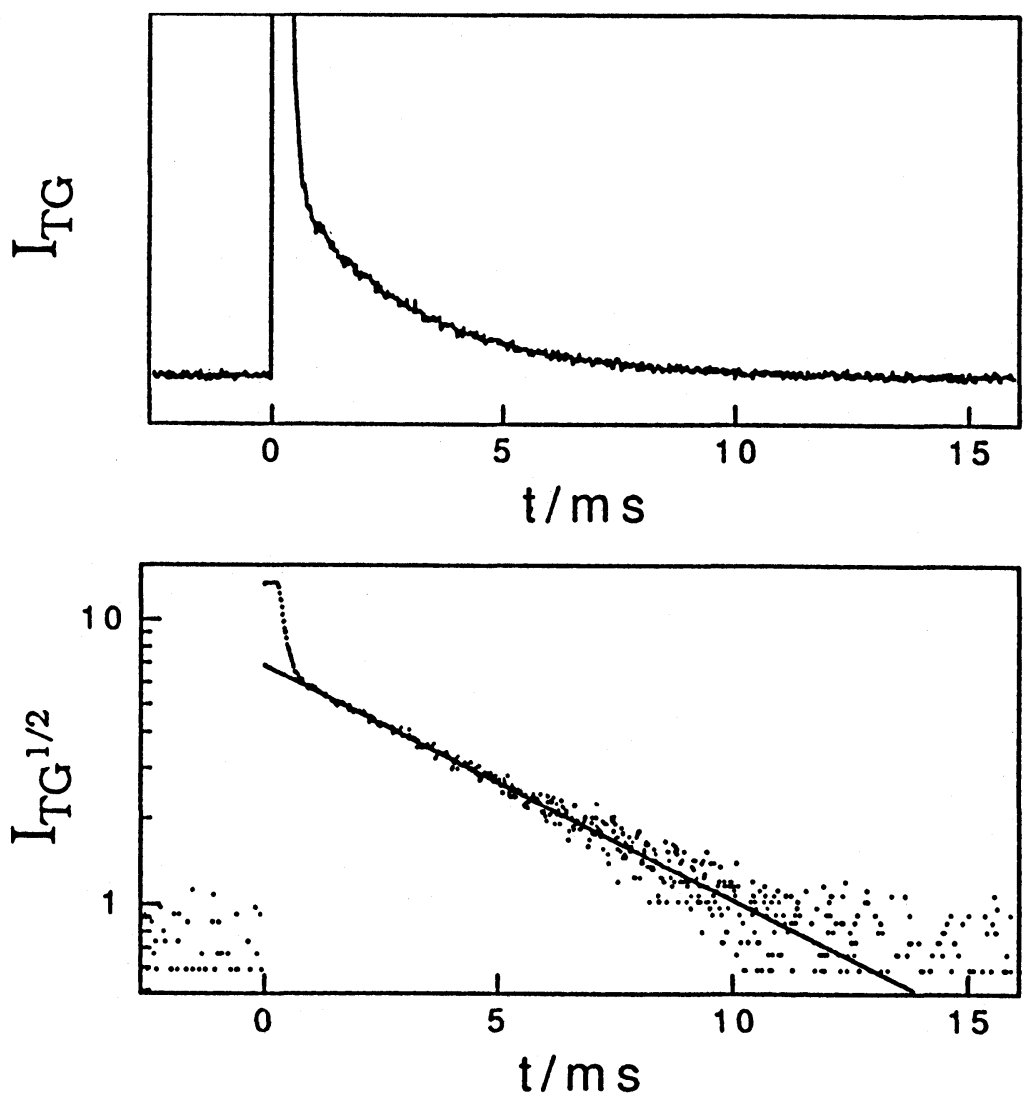

Figure 2 (a) The time dependence of the TG signal which represents the mass diffusion of MR in acetone at room temperature. (b) Semi-logarithm plot of the $\mathrm{I}_{\mathrm{TG}}^{\frac{1}{2}} \mathrm{p}^{\circ}$

dependence of the TG signal after the excitation of MR in acetone, and 1-butanol. In the early time region after the excitation, the TG signal due to the thermal grating dominates. This signal decays owing to the thermal diffusion and then the signal due to the population grating becomes apparent (Figure 2-a and 3-a). The TG signal intensity due to the population grating relaxes to the baseline level at a sufficiently long time. The decay rate of the TG signal does not vary even when the probe laser power is reduced to $\sim 1 / 10$ under our experimental conditions. Also we confirm that the decay rate does not depend on the repetition rate of the excitation laser from 4 $\mathrm{Hz}$ to $2 \mathrm{~Hz}$.

We should first know whether the TG signal due to the mass diffusion comes from the $\delta \mathrm{n}_{\mathrm{p}}$ term or the $\delta \mathrm{k}_{\mathrm{p}}$ term (or mixture of both) in order to analyze the data. For that purpose, we first determine $D_{\mathrm{th}} q^{2}$ at a certain crossing angle from the initial part of the signal by adjusting the data of $\sqrt{\mathrm{I}_{\mathrm{TG}}}$ with $\mathrm{a}^{*} \exp \left(-\mathrm{D}_{\mathrm{th}} \mathrm{q}^{2} \mathrm{t}\right)+\mathrm{b}(\mathrm{a}$ and $\mathrm{b}$ are adjustable constants). Then we try to see if $\mathrm{I}_{\mathrm{TG}}$ can be fitted by eq. (5) with $\delta \mathrm{k}_{\mathrm{p}}=$ 0 or $\delta \mathrm{k}_{\mathrm{p}} \neq 0$. Although the difference is subtle because of the minor contribution of 

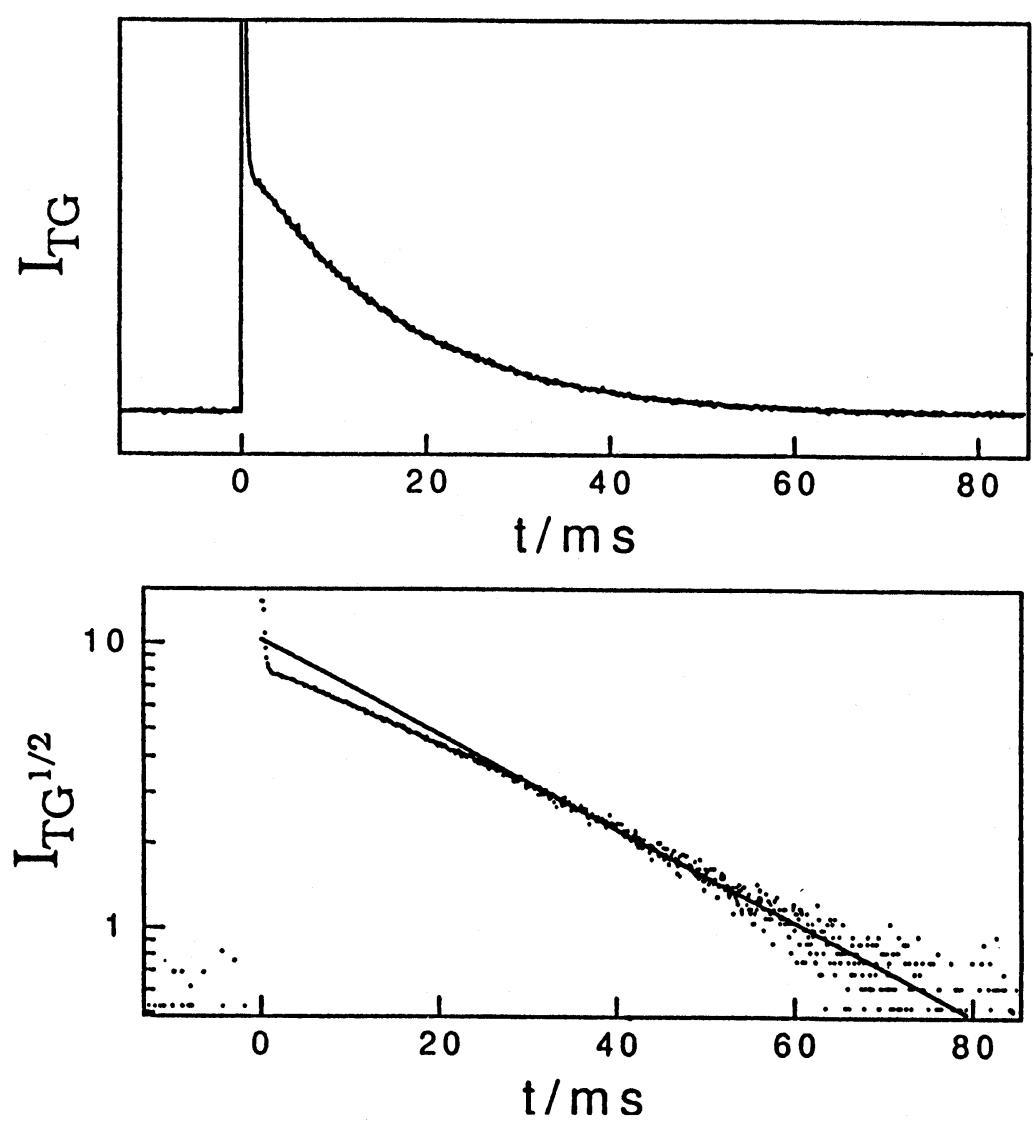

Figure 3 (a) The time dependence of the TG signal which represents the mass diffusion of MR in 1-butanol room temperature. (b) Semi-logarithm plot of the $\mathrm{I}_{\mathrm{TG}}^{\frac{1}{2}}$.

the population grating, we find eq. (5) with $\delta \mathrm{k}=0$ fits the data better. Therefore it is concluded that the TG signal which represents the mass diffusion mainly comes from the phase grating.

After the complete decay of the thermal grating signal, the square root of the population grating signal should decay bi-exponentially as predicted by eqs. (5) and (6a). The semi-logarithm plots of $\sqrt{\mathrm{I}_{\mathrm{TG}}^{(t)}}$ of the population grating signals are shown in Fig. 2-b and 3-b. In benzene, acetone, toluene, and diethylether solvents, it appears that the decays in solution are expressed well by a single exponential function, for example, as shown in Figure 2-b (Let us call these solvents as Type I solvents). The fact that the deviation from a single exponential decay is small indicates $D_{1} q^{2}$ $+k_{c t} \sim D_{c} q^{2}$ or $D_{t} \sim D_{c}$ since $k_{c t} \ll D_{c} q^{2}$ and $D_{t} q^{2}$. Then the general equation (6a) reduces to

$$
\delta n_{p}=[\Delta c]\left(\delta n_{c}-\delta n_{t}\right) \exp \left(-\left(D_{c} q^{2}+k_{c}\right) t\right)
$$


The pre-exponential factor should be negative because there is no observable dip between the thermal grating signal and the population grating signal. Since $\delta \mathrm{n}_{\mathrm{c}}$ and $\delta \mathrm{n}_{\mathrm{t}}$ are positive based on the previous discussion given in section 3 , we determine the relative magnitude of $\delta \mathrm{n}_{\mathrm{c}}$ and $\delta \mathrm{n}_{\mathrm{t}}$ as $\delta \mathrm{n}_{\mathrm{c}}<\delta \mathrm{n}_{\mathrm{t}}$. This relationship is considered to be reasonable because the absorption peak of the trans form is located at a longer wavelength than that of the cis form. ${ }^{9 a}$

On the other hand, in ethanol, 2-propanol, 1-butanol, 2-butanol and acetoaldehyde (We call type II solvents), $\log \left(\mathrm{I}_{\mathrm{TG}}(\mathrm{t})^{1 / 2}\right)$ vs $\mathrm{t}$ deviates slightly downward from the straight line calculated by the least square method from the data in the later time region as typically shown in Figure 3-b. The minor downward deviation from the single exponential fitting is considered to be due to the first term in eq. (6-a). Each of the time dependence and the downward deviation indicates that $\delta \mathrm{n}_{1}$ should be positive and $D_{c} q^{2}+k_{c t}<D_{t}$ Both conditions are satisfied under the condition $D_{c}<D_{t}$, although the difference should be very small. According to a calculation of the molecular volume of the cis and trans forms based on the molecular structure and the van der Waals radii, we find that the cis form has a slightly smaller molecular size than the trans form, which was also concluded by an independent group. ${ }^{8 a}$ Therefore, the above observation indicates that the smaller cis form of MR has a smaller diffusion coefficient than the larger trans form. Similar unexpected results with much larger differences have been observed in polymer hosts previously and it has been interpreted as due to a strong interaction between the cis isomer and the polymer hosts. $^{8 a}$ Our finding of the solvent dependence suggests that the interaction between the solvents and MR is likely due to the hydrogen bonding interaction. Probably the bended structure of the cis form is more easily affected by the hydrogen bonding with the solvents.

Unfortunately, $D_{c}$ and $D_{t}$ cannot be determined accurately even in the type II solvents, because of the small difference between $D_{c}$ and $D_{t}$ and also of the small contribution of the first term in eq. (2-a). We calculate the decay rate by the least square fitting of the relatively slow decay parts. The uncertainty due to this deviation from a single exponential decay is estimated to be less than $\pm 10 \%$. D obtained from the slopes of the k vs. $\mathrm{q}^{2}$ plots are listed in Table. The intercepts $\mathrm{k}_{\mathrm{ct}}$ are very small and this is consistent with long lifetimes of the cis form in dark (an order of seconds).

According to the Stokes-Einstein (SE) relation, the diffusion coefficient of a spherical molecule of radius $r$ in a continuum solvent of viscosity $\eta$ is given by, ${ }^{13,14}$

$$
D_{\mathrm{SE}}=\mathrm{kT} / \mathrm{f} \pi \eta \mathrm{r}
$$

The value of $f$ is 6 for the stick boundary condition of a spherical molecule. If the molecule is a prolate or oblate ellipsoid, $f$ should be corrected by the equation derived by Perrin. ${ }^{14}$ In Table, we also list the calculated D based on the Stokes-Einstein relation. These values, $\mathrm{D}_{\mathrm{SE}}$, are usually much or slightly smaller than those observed. The underestimate of D by the SE relation has been frequently reported, especially for relatively small solutes compared with the solvent size. ${ }^{15-18}$ Here we find that the differences are larger in type II solvents and it indicates that the molecular size is not the only cause of the difference. 
Table Translational diffusion coefficients of MR (D) in various solvents determined by the TG method, calculated eqs. $(10)\left(D_{s E}\right)$ and $(11)\left(D_{s w}\right)$. The unit of $D$ is $10^{-9} \mathrm{~m}^{2} \mathrm{~s}^{-1}$.

\begin{tabular}{llll}
\hline solvent & $D_{\mathrm{c}}\left(\sim D_{\mathrm{t}}\right)$ & $D_{\mathrm{SE}}$ & $D_{\mathrm{Sw}}$ \\
\hline benzene & 1.35 & 0.64 & 1.20 \\
acetone & 2.64 & 1.36 & 4.57 \\
toluene & 1.29 & 0.75 & 2.50 \\
diethylether & 3.41 & 1.85 & 7.59 \\
ethanol & 0.90 & 0.36 & 0.92 \\
1-butanol & 0.52 & 0.15 & 0.47 \\
2-butanol & 0.39 & 0.11 & 0.37 \\
2-propanol & 0.56 & 0.18 & 0.59 \\
acetoaldehyde & 2.61 & 1.97 & 7.00 \\
\hline
\end{tabular}

Molecular volumes of the solvents are calculated from the density and molecular weight with a constant free volume $(26 \%){ }^{20}$ The viscosities of the solvents are taken from reference 21 .

So far, many modifications of the SE theory have been proposed. For example, Spernol and Wirtz modified the SE equation by taking into account the finite size of the solvent molecule and rough interactive potential between solute and solvent by introducing an empirical microfriction factor $\mathrm{f}_{\mathrm{sw}}{ }^{19}$

$$
\begin{gathered}
\mathrm{D}_{\mathrm{SW}}=\mathrm{kT} / 6 \pi \eta r f_{\mathrm{SW}} \\
\mathrm{f}_{\mathrm{SW}}=\left(0.16+0.4 \frac{\mathrm{r}_{\mathrm{A}}}{\mathrm{r}_{\mathrm{B}}}\right)\left(0.9+0.4 \mathrm{~T}_{\mathrm{A}}^{\mathrm{r}}-0.25 \mathrm{~T}_{\mathrm{B}}^{\mathrm{r}}\right)
\end{gathered}
$$

where $r_{A}$ and $r_{B}$, respectively, represent the radii of the solute $\left(r_{A}\right)$ and the solvent $\left(r_{B}\right)$. Further, the reduced temperature, $T_{A}^{r}$ and $T_{B}^{r}$ are calculated by

$$
\mathrm{T}_{\mathrm{X}}^{\mathrm{r}}=\left(\mathrm{T}-\mathrm{T}_{\mathrm{X}}^{\mathrm{f}}\right) /\left(\mathrm{T}_{\mathrm{X}}^{\mathrm{b}}-\mathrm{T}_{\mathrm{X}}^{\mathrm{f}}\right)
$$

using the freezing $\left(\mathrm{T}_{\mathrm{X}}^{\mathrm{f}}\right)$ and boiling $\left(\mathrm{T}_{\mathrm{X}}^{\mathrm{b}}\right)$ points of the solute $(\mathrm{X}=\mathrm{A})$ or the solvent $(\mathrm{X}=\mathrm{B})$. This correction method has been recommended by several groups. ${ }^{1718}$ The calculated D based on this equation are listed in Table. Generally D in alcoholic solvents agree well with the calculated $\mathrm{D}_{\mathrm{sw}}$. However $\mathrm{D}_{\mathrm{sw}}$ in the type I solvents and aldehyde give overestimates of $\mathrm{D}$.

\subsection{Diffusion of transient radicals in solutions}

As an example of the diffusional dynamics of a transient radical in solution, we choose pyrazinyl radical created from pyrazine by the photoinduced hydrogen abstraction reaction. ${ }^{22}$ After the excitation of pyrazine in 2-propanol at $308 \mathrm{~nm}$ under nitrogen saturated condition a strong TG signal is observed. This signal first decays for a period of an order of $\mu \mathrm{s}$ and the origin of the signal is assigned to the thermal grating. We cannot observe any slow rising component in the early part of the thermal grating signal, which indicates that the triplet state of pyrazine is efficiently quenched by 2-propanol. This thermal grating signal decays down to the baseline once, rises to a certain intensity and then decays again to the baseline (Figure 4). Apparently 


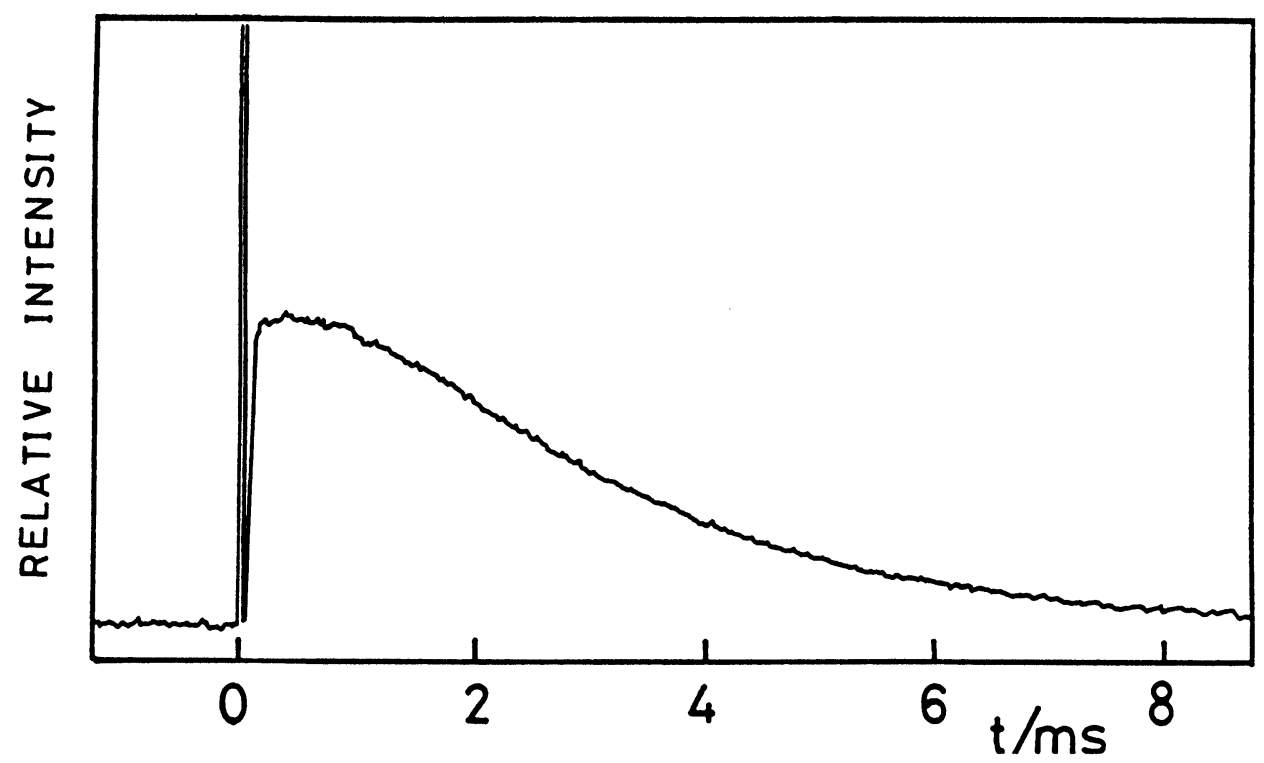

Figure 4 (a) Typical time dependence of the TG signal which represents the mass diffusion of pyrazine in 2-propanol at room temperature. The initial sharp spike-like signal is due to the contribution of the thermal grating.

the time developments of these slow components are governed by the mass diffusion in solution. The fact that the thermal grating signal drops down to the baseline once indicates that the $\delta \mathrm{k}_{\mathrm{p}}$ term in eq. (5) is negligible and the main contribution of the $\delta \mathrm{n}_{\mathrm{p}}$ term is positive. In other words, the absorption of the chemical species at the probe wavelength is negligible and this is confirmed by the transient absorption measurement. Fig. 5 shows the $\log \left(\mathrm{I}_{\mathrm{TG}}\right)^{1 / 2}$ vs $t$ plot. After the thermal grating the TG signal is well expressed by two exponentials with different signs of the pre-exponential factors.

$$
\left(I_{T G}\right)^{1 / 2}=a_{s} \exp \left(-k_{s} t\right)+a_{\mathrm{f}} \exp \left(-k_{\mathrm{f}} \mathrm{t}\right)
$$

where subscripts $s$ and $f$ stand for the slow and fast components, respectively and $a_{\mathrm{f}}<0<\mathrm{a}_{\mathrm{s}},\left|\mathrm{a}_{\mathrm{s}}\right|>\left|\mathrm{a}_{\mathrm{f}}\right|$. When an air saturated solution is used as the sample, the slow component decays with a faster time constant.

We identify the diffusing species which contribute in the TG signal based on the following facts.

(1) The slow decay component decays faster under the air saturated condition. Since it is unlikely that dissolved nitrogen or oxygen change the diffusion process in solution, the slower decay must be due to the quenching of the diffusing species by oxygen.

(2) Pyrazine is already known to create pyrazinyl radical in 2-propanol solution by light irradiation from the photoexcited triplet state. ${ }^{23,24}$ 


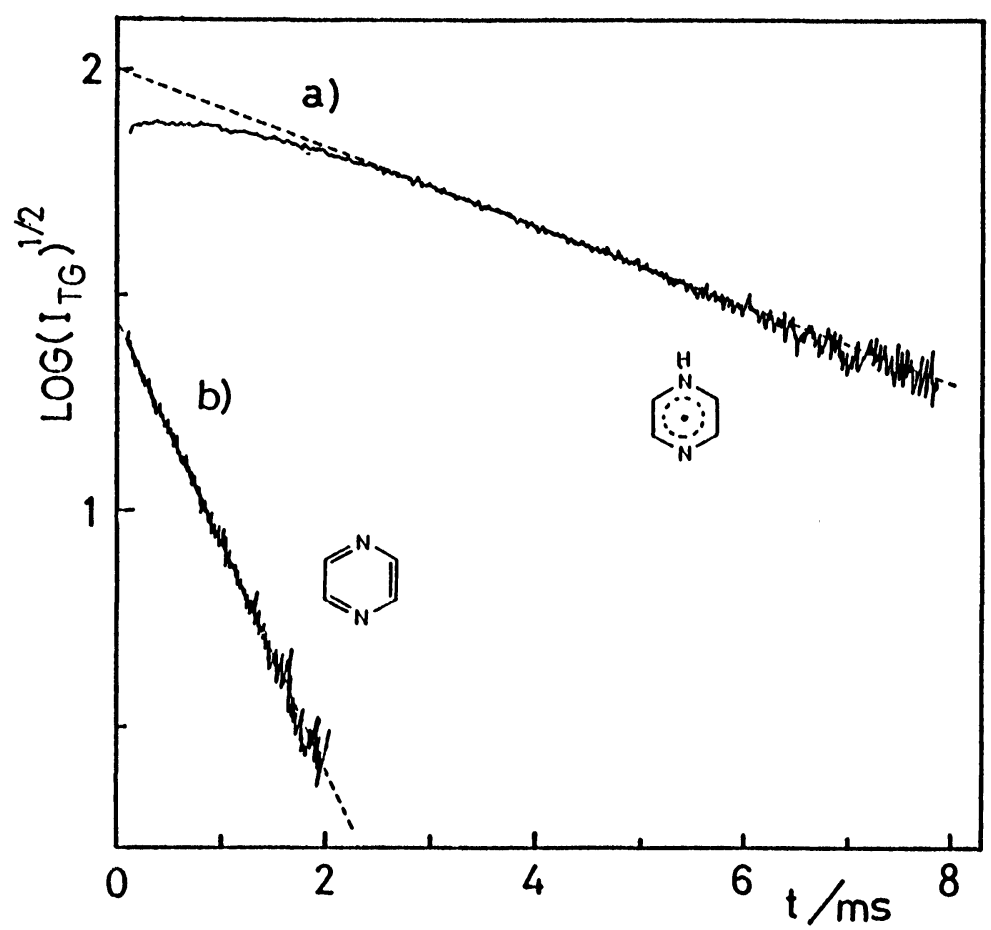

Figure 5 (a) $\log \left(\mathrm{I}_{\mathrm{TG}}\right)^{1 / 2}$ vs $t$ plot of the TG signal of Figure 4. Broken line represents the least square fit of the slow component. (b) solid line represents the difference between the solid and broken lines of (a). The broken line represents the least-square fit of the rise component.

$$
{ }^{3} \mathrm{Py}+\left(\mathrm{CH}_{3}\right)_{2} \mathrm{CHOH} \longrightarrow \mathrm{PyH}+\left(\mathrm{CH}_{3}\right)_{2} \mathrm{COH} \text {. }
$$

(3) The created radicals as well as the parent molecules (pyrazine and 2-propanol) possess their absorption bands at shorter wavelengths than that of $\mathrm{He}-\mathrm{Ne}$ laser. ${ }^{23,25}$ Therefore all of $\delta n_{i}$ and $\delta n_{i}$ should be positive.

(4) Pyrazine has much stronger absorption bands in the near UV region compared with that of 2-propanol. Therefore the depletion of pyrazine should form a larger phase grating than 2-propanol. ${ }^{26}$

(5) The transient absorption measurement shows a strong absorption band near 350 $\mathrm{nm}$ at $200 \mu \mathrm{s}$ after the laser irradiation. ${ }^{22}$ The shape of the absorption band is similar to that of the pyrazinyl radical and there is no trace of absorption band which resembles that of the 2-hydroxypropyl radical. ${ }^{23,25}$

(6) The TG experiment on acetone/2-propanol, ${ }^{27}$ which produces the 2hydroxypropyl radical exclusively, shows that the TG signal is relatively weak and the diffusional motion of this radical is much faster than the species which shows the slow decay component in the TG signal of pyrazine/2-propanol. 
Considering these aspects, we assign the species which gives the slow rising component to pyrazine and which gives the slow decay component to the pyrazinyl radical. Probably 2-propanol and 2-hydroxypropyl radical cannot be detected because of the weak absorption coefficients. Figure 6 shows the $q^{2}$ dependence of $k_{s}$ and $k_{f}$. According to the above assignment, the slopes for $k_{s}$ and $k_{f}$ represent $D$ of the pyrazinyl radical and the 2-hydroxypropyl radical, respectively. The obtained $\mathrm{D}$ are $1.3 \times 10^{-9} \mathrm{~m}^{2} / \mathrm{s}$ for pyrazine and $3.1 \times 10^{-10} \mathrm{~m}^{2} / \mathrm{s}$ for the radical. Both plots seem to have near zero intercepts within the experimental error.

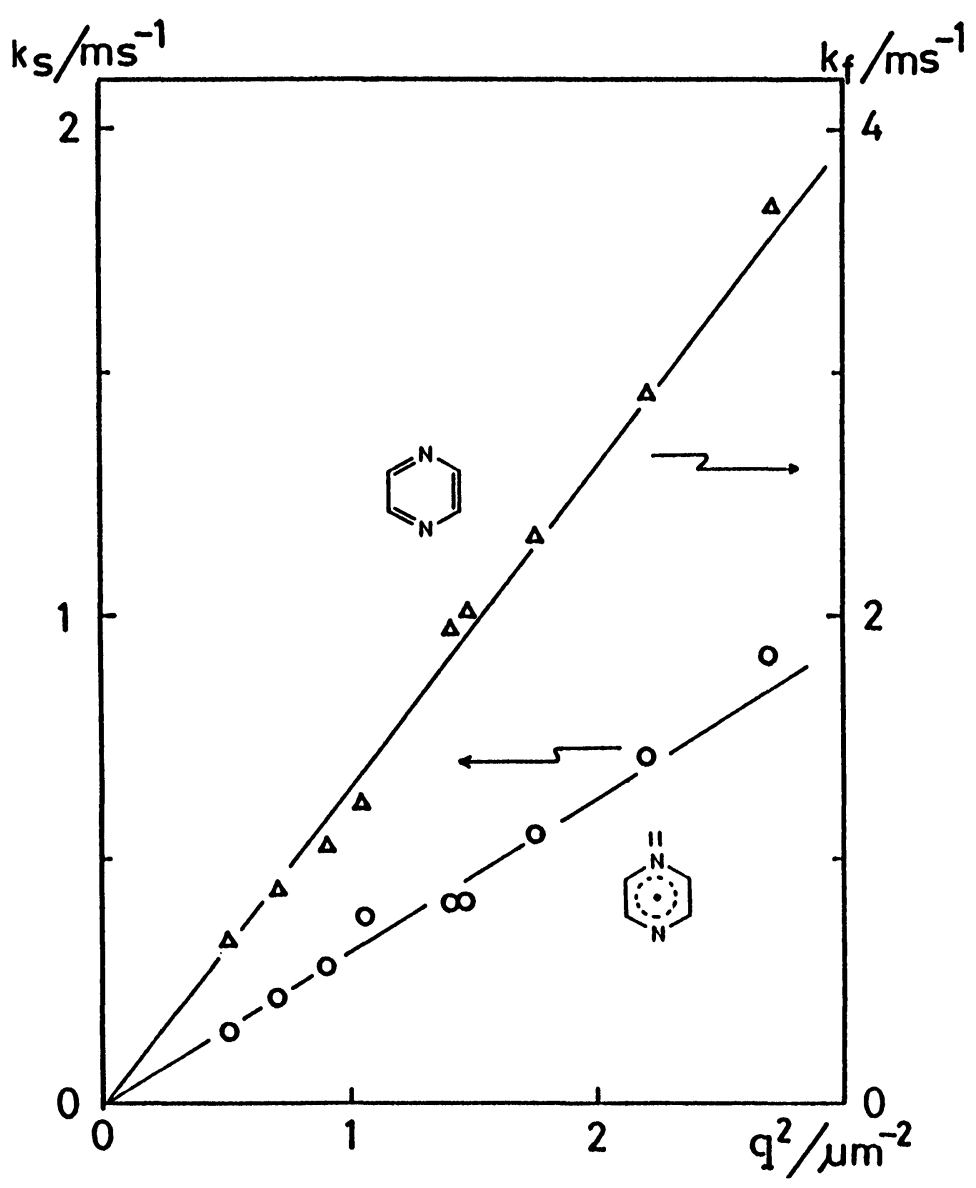

Figure 6 Plot of the decay rate constants, $k_{s}$ (circles) and $k_{f}$ (triangles) vs. $q^{2}$ at room temperature for pyrazine/2-propanol.

A striking feature of the above result is the very small $\mathrm{D}$ value of the transient radical (pyrazinyl radical) compared with the parent molecule (pyrazine), even though 
the molecular volume should not be different so much (the difference is the only one hydrogen atom on the nitrogen atom of pyrazine). In other photoinduced hydrogen abstraction reaction systems, such as phenazine, benzoquinone, and benzophenone in alcoholic solvents, similar time dependence of the TG signal are observed ${ }^{27}$ Based on similar analyses as that in the pyrazine case, we find that the radical diffusions are generally 3 4 times slower than the parent molecules with similar molecular volumes. Therefore we believe that we are observing a general phenomenon at least in the hydrogen abstraction reaction systems.

If we only consider the hydrodynamic interaction for the dynamics of these species in solution, the diffusion is expressed by the SE (eq. (10)) or modified SE (eq. (11)) equations. Obviously, the SE relation (eq. (10)) does not explain the difference in $\mathrm{D}$ between the radicals and the parent molecules. Although we do not know the reduced temperature $T_{A_{r}}$ of the radicals in eq. (11), it cannot be increased so much to give 4 times smaller $\mathrm{D}$ values. Therefore we must conclude that a specific attractive radical-solvent interaction is involved in the diffusion process of the radicals. We are now continuing the investigation of the diffusion processes of transient radicals in other photochemical reaction systems and in other solvents.

\subsection{TG method for probing the molecular dynamics in solutions}

Here we briefly comment on the advantages listed in the introduction from the experimental point of view.

1. The high sensitivity of the TG method enables us to study the solution dynamics with a very dilute concentration of a tracer. It ensures that the observing dynamics is truly controlled by the solvent (the tracer does not affect the motion of the solvent) and that the solute-solute interaction can be neglected.

2. Together with the advantage 1 , the diffusion process of a trace amount of sample which is very difficult to be obtained such as biological molecules can be investigated.

3. The merit that the method is less time consuming, has been frequently utilized for measuring $D$ in polymer solution ${ }^{8}$ or polymer glasses. ${ }^{9}$ Also we expect that the dynamics of biological macromolecules in solution can be studied by the TG method even though the movements of these macromolecules are very slow and it takes a long time to be measured by the conventional techniques. ${ }^{13}$

4. The investigations of the dynamics in liquid crystals ${ }^{11}$ demonstrate the above advantage beautifully. By choosing the fringe direction, the thermal diffusion coefficients as well as the mass diffusion coefficients parallel to and perpendicular to the director have been measured and discussed.

5. and 6. Reports on the comparison of the D values measured by the TG method with those measured by other conventional methods such as the tracer diffusion, NMR, light scattering methods, are still rare. Further, as far as we know, there is no report on the comparison of the D values measured by the TG method and by the fluorescence quenching method, which is suited to study short lived species? Therefore we do not know if the assumptions made on the quenching process that 
are made implicitly in the analysis are adequate. Comparing the results with those obtained by the TG method, we might have some insight on the quenching process.

7. This is a very unique advantage of this method. As far as we know, there is no report concerning this point. However using a similar technique, fluorescence recovery after fringe-pattern photobleaching method, Ott et al. have observed an anomalous diffusion, distance-dependent $\mathrm{D}$ in a living polymer. ${ }^{28}$ Recently we have observed a similar distance-dependent diffusion process in a polymer solution system. ${ }^{29}$ We believe that in the near future this advantage will attract many investigators working on the solution dynamics and polymer dynamics.

Even though there are many unique attractive points in the TG method, we should admit there are several disadvantages.

(i) The first and most serious disadvantage is that the TG method cannot be applied to chemically stable molecules because the modulation of the optical properties cannot be created. One possible way to overcome this disadvantage is that we might be able to use a long lived metastable state such as the excited triplet state as the source of the grating. If one can detect the difference of the optical properties between the ground state and the excited triplet state, the TG method can be used for many molecules as long as the quantum yield of the triplet formation is reasonablly high.

(ii) The TG signals of MR and pyrazine/2-propanol systems are dominated by the phase grating. In this case, the analysis of the TG signal is relatively simple. On the other hand, if both $\delta \mathrm{n}_{\mathrm{p}}$ and $\delta \mathrm{k}_{\mathrm{p}}$ terms cannot be neglected, extraction of the decay rate constants becomes very difficult. The difficulty increases more when many chemical species are involved in the signal. In these cases, changing the probe beam wavelength might help to simplify the analysis of the TG signal.

\section{Summary}

Some examples of investigating the molecular dynamics in solution by the TG method are demonstrated. First, MR is used for probing the solution dynamics in a number of organic solvents. Diffusion coefficients of methyl red in organic solutions can be measured based on the interpretation of the TG signal; contribution of the phase grating is dominant in the TG signal. The cis form, which has a smaller volume possesses smaller $\mathrm{D}$ than that of the trans form. This result is attributed to the hydrogen bonding interaction between MR and the solvents. The diffusion coefficients of MR are much larger than those calculated by the Stokes-Einstein equation in all the solvents we examined. The experimentally obtained $\mathrm{D}$ at room temperature in alcoholic solvents agree well with the values calculated by the modified StokesEinstein equation according to Spernol and Wirtz. It is shown that the TG technique is a convenient means to study the translational diffusion process in solution.

As a unique application of the TG method, D of transient radicals created by photochemical reactions are investigated. For example, in the pyrazine/2-propanol case, the signal consists of a fast decay due to the thermal diffusion, a slow rise due to the diffusion of pyrazine and another slow decay due to the diffusion of the pyrazinyl radical. The translational motion of the transient radicals we examined are 
generally several times slower than those of the parent non-radical molecules. The advantages and disadvantages of the TG method for studying the solution dynamics are discussed.

\section{Acknowledgement}

This work is supported by Scientific Research Grant-in-Aid for the Priority Area of "Molecular Approaches to Nonequilibrium Processes in Solution" and of "Molecular Magnetism" (Area No.1228/04242102) by the Ministry of Education, Science and Culture.

\section{References}

1. H. J. Eichler, P. Günter, D. W. Pohl. Laser Induced Dynamic Gratings, Springer-Verlag, Berlin, 1986.

2. M. Terazima, N. Hirota. J. Chem. Phys., 95, 6490 (1991); Chem. Phys. Lett., 189, 560 (1992).

3. (a) A. Miller, D. A. B. Miller, S. D. Smith. Adv. Phys., 30, 697 (1981).

(b) R. K. Jain, Opt. Eng., 21, 199 (1982).

4. (a) K. A. Nelson, M. D. Fayer. J. Chem. Phys., 72, 5202 (1980).

(b) Y-X. Yan, L-T. Cheng, K. A. Nelson. J. Chem. Phys., 88, 6477 (1988).

(c) S. M. Silence, A. R. Duggal, L. Dhar, K. A. Nelson. J. Chem. Phys., 96, 5448 (1992).

5. (a) F. W. Deeg, S. R. Greenfield, J. J. Stankus, V. J. Newell, M. D. Fayer. J. Chem. Phys., 93, 3503 (1990).

(b) R. S. Moog, M. D. Edlger, S. G. Boxer, M. D. Fayer. J. Phys. Chem., 86, 4694 (1982).

6. (a) X. R. Zhu, J. M. Harris. Chem. Phys., 124, 321 (1988); 157, 409 (1991).

(b) F. W. Deeg, J. Pinsl, C. Bräuchle. J. Phys. Chem., 90, 5710 (1986).

(c) R. K. Grygier, P. A. Brugger, D. M. Burland. J. Phys. Chem., 89, 112 (1985).

7. (a) W. R. Ware, T. L. Nemzek. Chem. Phys. Lett., 23, 557 (1973).

(b) T. L. Nemzek, W. R. Ware. J. Chem. Phys., 62, 477 (1975).

(c) T. W. Scott, C. Doubleday Jr., Chem. Phys. Lett., 178, 9, (1991).

(d) S. Nishikawa, T. Asahi, T. Okada, N. Mataga, T. Kakitani. Chem. Phys. Lett., 185, 237 (1991).

8. (a) C. H. Wang, J. L. Xia. J. Phys. Chem., 96, 190 (1992).

(b) J. A. Lee, T. P. Lodge. J. Phys. Chem., 91, 5546 (1987).

(c) T. P. Lodge, J. A. Lee, T. S. Frick. J. Polym. Sci., 28B, 2607 (1990).

(d) J. L. Xia, S. S. Gong, C. H. Wang. J. Phys. Chem., 91, 5805 (1987).

(e) J. L. Xia, C. H. Wang, J. Chem. Phys., 88, 5211 (1988).; ibid, 92, 2603 (1990).

(f) Q. Tran-Cong, T. Chang, C. C. Han, Y. Nishijima. Polymer, 27, 1705 (1986); Q. Tran-Cong, T. Chang, C. C. Han, Polymer, 29, 2261 (1988).

(g) D. J. Miles Jr., P. D. Lamb, K. W. Rhee, C. S. Johnson Jr. J. Phys. Chem., 87, 4815 (1983).

(h) K. W. Rhee, D. A. Gabriel, C. S. Johnson Jr., C. S. J. Phys. Chem., 88, 4010 (1984).

9. (a) F. Rondelez, H. Hervet, W. Urbach. Chem. Phys. Lett., 53, 138 (1978).

(b) S. S. Gong, D. Christensen, J. Zhang, C. H. Wang. J. Phys. Chem., 91, 4505 (1987).

(c) J. Zhang, B. K. Yu, C. H. Wang. J. Phys. Chem., 90, 1299 (1986).

10. J. H. Wang, H. Takezoe, H. Yu, S. P. Chen. J. Appl. Phys., 53, 6513 (1982).

11. (a) H. Hervet, W. Urbach, F. Rondeley. J. Chem. Phys., 68, 2725 (1978).

(b) H. Takezoe, S. Ichikawa, A. Fukuda, E. Kuze. E. Jpn. J. Appl. Phys., 23, L78 (1984).

(c) M. Hara, H. Tenmei, S. Ichikawa, H. Takezoe, A. Fukuda Jpn. J. App. Phys., 24, L777 (1985).

(d) M. Hara, H. Takezoe, A. Fukuda Jpn. J. Appl. Phys., 23, 1420 (1984); ibid., 25, 1756 (1986).

12. M. Terazima, K. Okamoto, N. Hirota. J. Phys. Chem., submitted.

13. E. L. Cussler, "Diffusion", Cambridge University Press, Cambridge (1984).

14. F. Perrin. J. Phys. Radium., 7, 1 (1931).

15. T. Tominaga, S. Matsumoto. Bull. Chem. Soc. Jpn., 63, 533 (1990).

16. D. F. Evans, T. Tominaga, H. T. Davis. J. Chem. Phys., 74, 1298 (1981).

17. H-H. Schuh, H. Fisher. Helv. Chimi. Acta, 61, 2130 (1978). 
18. H. S. Sandhu, J. Mag. Res., 17, 34 (1975).

19. A. Spernol, K. Wirtz, Z. Natureforsch. 8a, 522 (1953).

20. J. T. Edward. J. Chem. Edc., 47, 261 (1970).

21 D. S. Viswanath, G. Natarajan, "Data Book on the Viscosity of Liquids", Hemisphere Co., New York (1989).

22. M. Terazima, N. Hirota. J. Chem. Phys., in press.

23 P. N. Moorthy, E. Hayon. J. Phys. Chem., 78, 2615 (1974). D. V. Bent, E. Hayon, P. N. Moorthy. J. Am. Chem. Soc., 97, 5065 (1982).

24. S. Basu, K. A. McLauchlan, G. R. Sealy. Chem. Phys. Lett., 88, 84 (1982). S. Yamauchi, N. Hirota. J. Phys. Chem., 88, 4631 (1984).

25. G. Porter, S. K. Dogra, R. O. Loutfy, S. E. Sugamori, R. W. Yip. J. Chem. Soc., Farad. Trans., I, $1462(1973)$.

26. R. S. Lonhurst, "Geometrical and Physical Optics", 2nd ed., Wiley, New York (1967).

27. M. Terazima, K. Okamoto, N. Hirota. to be submitted.

28. A. Ott, J. P. Bouchaud, D. Langevin, W. Urbach. Phys. Rev. Lett., 65, 2201 (1990).

29. M. Terazima, N. Hirota, unpublished results. 